\title{
Implementation of Novel Technique for Selective Harmonic Elimination in Multilevel Inverters Based on ICA
}

\author{
Ali Ajami, Mohammad Reza Jannati Oskuee, and Ata Ollah Mokhberdoran \\ Electrical Engineering Department, Azarbaijan Shahid Madani University, $35 \mathrm{Km}$ from Tabriz-Maragheh Motorway, \\ P.O. Box 53714-161, Tabriz, Iran \\ Correspondence should be addressed to Ali Ajami; ajami@azaruniv.edu
}

Received 4 April 2013; Accepted 27 May 2013

Academic Editor: C. M. Liaw

Copyright (C) 2013 Ali Ajami et al. This is an open access article distributed under the Creative Commons Attribution License, which permits unrestricted use, distribution, and reproduction in any medium, provided the original work is properly cited.

\begin{abstract}
Eliminating the specific harmonics especially low-order harmonics of the output voltage of 9-level inverter using SHE-PWM control scheme is investigated. Harmonic minimization is the intricate optimization problems because the nonlinear transcendental equations have multiple local optima. Increasing the degrees of freedom in the suggested method means that the number of switching angles increases. The suggested method is able to eliminate high number of undesired harmonics. As the number of switching angles increases, using either traditional iterative techniques or resultant theory method gets useless. In this paper to overcome this problem the imperialist competitive algorithm (ICA) is used. Also a DC-DC converter is used to enhance the SHE performance in the range for which the conventional SHE methods do not have any solution. Experimental and simulation results of a 9-level inverter show that the proposed method effectively minimizes a large number of particular harmonics so the total harmonics distortion of output voltage will be lower.
\end{abstract}

\section{Introduction}

THE developments of FACTS devices [1, 2], medium voltage drives $[3,4]$, and various types of renewable energy resources $[5,6]$ have given great opportunities for the implementation of medium- and high-power inverters. The main problem with these applications is the frequency constraint of the pulse width modulation (PWM) which are limited by switching losses and electromagnetic interferences which is the results of high $d v / d t$. Thus, to overcome the mentioned problems, selective-harmonic-elimination- (SHE-) based optimal pulse width modulation (OPWM) are proposed which are able to reduce the switching frequency and the total harmonic distortion of output voltage [7]. A typical multilevel inverter utilizes several DC voltage sources to provide a stepwise waveform in output voltage which makes a great development on output voltage THD while the output waveform approaches nearly sinusoidal waveform [8]. Related to the inverter circuit topologies the dc sources can be interconnected or isolated [7]. Because of the intricacy of the problem, in most studies on the SHE methods for multilevel inverters, it is assumed that only one switching angle per each voltage level is defined and the dc voltage sources are balanced (equal to each other). But in practical applications, depending on the output waveform and operation scheme of the inverter, the dc sources could be unbalanced or several switchings per each level are involved [9-13]. SHE method is a modulation strategy whose goal is to determine the proper switching angles to eliminate the number of low-order harmonics which cause to minimize the output waveform THD [14]. The SHE method requires low switching frequency and stepwise waveform of output voltages to be applied [15]. The main goal in SHE method is to determine the switching angles in which with the obtained switching angles the fundamental component reaches to the desired value and the undesired harmonics; basically low-order harmonics are eliminated [16]. The defined objective function for SHE problem includes a set of nonlinear transcendental equations which may involve several local optima. Solving the SHE problem is available with the help of several procedures. Resultant theory is a novel which is based on methodical calculations [17]. In resultant theory method the provided equations which are defined for SHE are converted into an equivalent set of polynomial equations, and then resultant theory is applied to the obtained equivalent equations which are naturally highorder polynomials. The main problem with this method is 


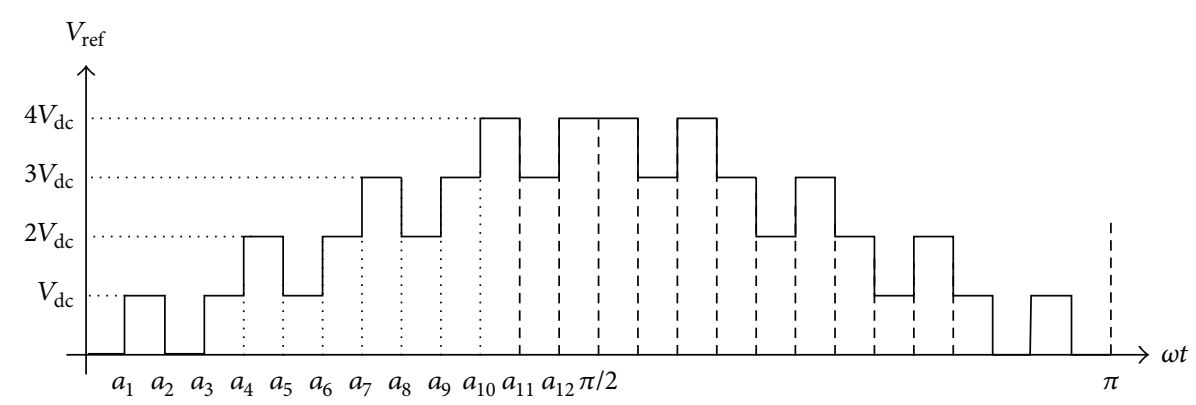

FIGURE 1: Half cycle of the reference phase voltage of a 9-level inverter.

the complexity of calculation. The complexity increases when the number of switching angles is increased and so makes the equivalent equation harder to be solved or even not to be solved. Another approach for solving is the NewtonRaphson method, and it is a Numerical iterative technique [18]. However, the great difficulty of these techniques is the proper initial guess requirement that should be near to the exact solution. It is evident that giving a proper guess is very difficult in most cases but if a proper initial guess is available, Newton-Raphson method works properly. This difficulty is the result of the SHE problems search space which is unknown for anybody, and no one knows whether a solution exists or not, and if exists, what is the proper initial guess? A recently developed novel method to deal with the SHE problem is based on evolutionary algorithms such as Bee algorithm [19]. However the search spaces intricacy would increase vividly if the number of switching angles increases and both methods fall into the trap of local optimum points of search space. Surely, the precise limitation for the number of switching angles cannot be determined in evolutionary algorithms. So, increasing the number of switching angles reduces the probability of finding the optimum switching angles, unfortunately. The SHE-PWM is a novel method that provides more number of the degrees of freedom (DOFs) and makes available to eliminate more harmonic components with no need to change the perceivable hardware of the inverter [20, 21]. In SHE-PWM, each active device can be switched at least twice per cycle, and larger number of harmonic components than in the case of fundamental frequency switching scheme can be eliminated. The major problem related to the SHE-PWM method is that as the number of switching angles is increased; none of aforementioned methods can be applied to determine the switching angles. In this paper, a novel method based on ICA is developed to deal with the SHE-PWM problem. Simulation and experimental results are obtained for a 9-level cascaded multilevel inverter to validate the efficiency of the proposed method and accuracy of the authors. The rest of this paper is organized as follows.

Section 2 describes the multilevel inverters output voltage and its harmonic spectrum. Section 3 defines the SHE approach objective.

In Section 4, the obtained simulation results are presented. Also to improve the capability of the SHE, an efficient solution is presented. Finally, in Section 5 experimental results are provided to confirm the author's accuracy. Section 6 is specified for the conclusion.

\section{Output Voltage of Multilevel Inverter}

A half cycle of a typical waveform of the reference phase voltage of a 9-level inverter synthesized by several DC sources using SHE-PWM method is illustrated in Figure 1.

The $a_{1}-a_{12}$ are the required switching angles to specify the whole cycle of the shown waveform. Regarding Figure 1 the reference voltage, $V_{\text {ref }}$, can be demonstrated in terms of step function $u(\omega t)$.

For positive half of the waveform:

$$
\begin{array}{r}
V_{a p}=u\left(\omega t-a_{1}\right)-u\left(\omega t-a_{2}\right)+u\left(\omega t-a_{3}\right)+\cdots \\
+u\left(\omega t-a_{10}\right)-u\left(\omega t-a_{11}\right)+u\left(\omega t-a_{12}\right) ; \\
0<\omega t<\pi .
\end{array}
$$

The negative half is in the opposite direction and lagging $V_{a p}$ in $\pi$ radians:

$$
V_{a n}=-V_{a p}(\omega t-\pi) ; \quad \pi<\omega t<2 \pi .
$$

Therefore the sum of (1) and (2) affords the reference phase voltage illustrated in

$$
V_{a}=V_{a p}+V_{a n}
$$

To identify instantaneous value of reference phase voltage the Fourier analysis is applied and the following expression is obtained. If the presented waveform in Figure 1 was considered for one cycle, it can be seen that it is an odd function and so it will contain only odd-order harmonics:

$$
\begin{aligned}
V_{a}=\sum_{n=1}^{\infty} \frac{4}{n \pi} V_{\mathrm{dc}} \sin \left(\frac{n \pi}{2}\right) \\
\quad \times\left(\cos \left(n a_{1}\right)-\cos \left(n a_{2}\right)+\cos \left(n a_{3}\right)\right. \\
+\cos \left(n a_{4}\right)-\cos \left(n a_{5}\right)+\cos \left(n a_{6}\right) \\
+\cos \left(n a_{7}\right)-\cos \left(n a_{8}\right)+\cos \left(n a_{9}\right) \\
\left.+\cos \left(n a_{10}\right)-\cos \left(n a_{11}\right)+\cos \left(n a_{12}\right)\right) \sin (n \omega t) .
\end{aligned}
$$




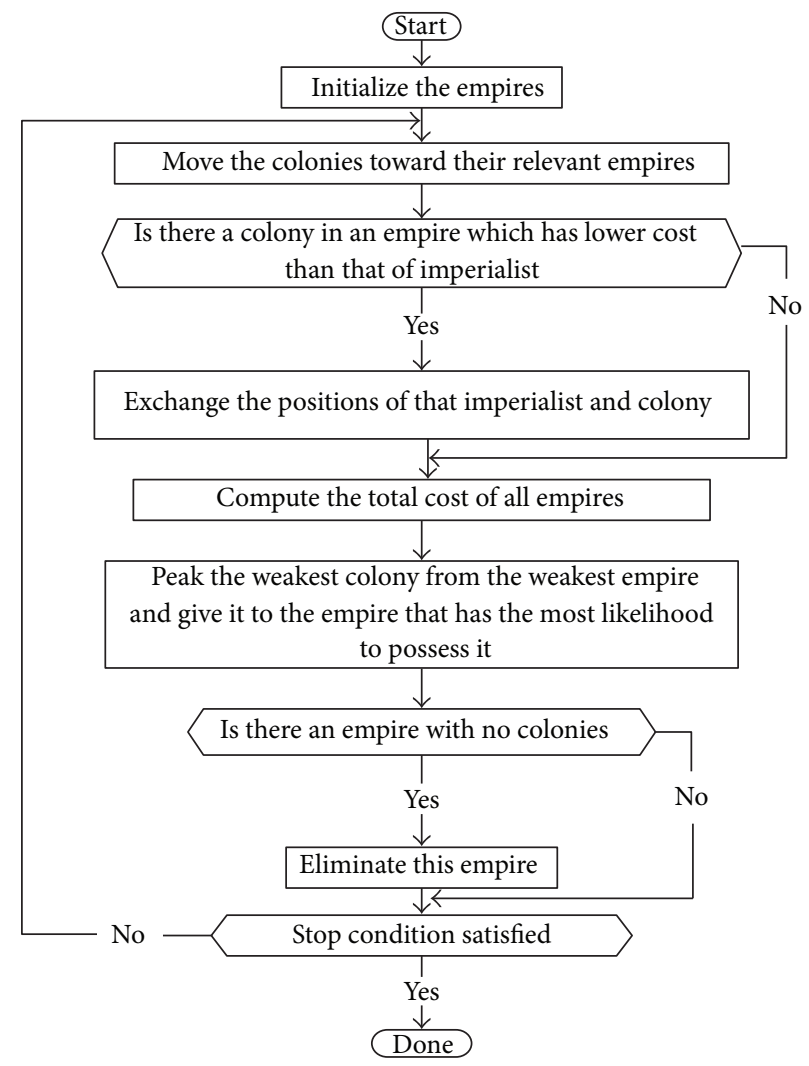

FIGURE 2: The flowchart of ICA process.

In (4), the negative signs indicate the falling edges of the staircase voltage waveform and vice versa. The objective of SHE-PWM method is aimed to choose the set of switching angles $a_{1}-a_{12}$ such that the identified lower-order harmonics are suppressed, and, at the same time, the amplitude of fundamental component becomes equal to the desired value.

\section{Selective Harmonic Elimination}

In most papers, the staircase voltage waveform is considered in SHE method in multilevel inverters $[22,23]$. In this case, for a $2 s+1$-level inverter, the number of switching in a quarter of cycle is limited to $s$, where $s$ is the number of individual DC sources. So the number of harmonics which can be eliminated from the output voltage is $s-1$. In order to increase the DOFs and eliminating more harmonics than the case of fundamental frequency switching scheme without any manipulation on inverters hardware, the SHE-PWM technique is proposed in [12], which is also denominated virtualstage PWM. This technique is one of the powerful theories that applies to multilevel inverters in order to generate high-quality voltage waveform with less switching frequency in comparison with other PWM methods. The generalized formula to eliminate lower-order harmonics in multilevel inverter with nonequal dc sources is also suggested in [21]. Commonly, the number of switching can vary in different levels. However, for simplicity, the number of switching is evaluated to be equal in this paper for different levels. Figure 1 presents the output voltage of 9-level inverter for three times of switching at each level. If $k$ indicates the number of switching at each level, switching frequency of the SHE-PWM scheme will be $k$ times the fundamental frequency. Therefore, the number of harmonic components that can be eliminated from the output voltage is evaluated by $k \times s-1$ for the 9 level. For $k=3$, the output voltage that is shown in Figure 1 , there are 12 DOFs, and so 11 undesired harmonics can be eliminated from the output voltage of inverter. The SHE PWM method is able to eliminate the nontriple lower-order harmonics up to $3 k s-2$ when $k s$ is odd and up to $3 k s-1$ order harmonic while $k s$ is even. It is noticeable that eliminating the triple harmonics is not necessary for three-phase applications since these harmonics should automatically be removed from the line-to-line voltage. A 9-level inverter is defined as a case study to assess the impact of SHE-PWM on its harmonic spectrum. Objective function for a 9-level inverter is the result of combination of 12-nonlinear equations that one equation is for fundamental component and the other equations are related to the undesired harmonics.

The mentioned equations are presented as follows:

$$
\begin{aligned}
\left(V_{a}\right)_{1}=\frac{4}{\pi} V_{\mathrm{dc}}( & \cos \left(a_{1}\right)-\cos \left(a_{2}\right)+\cos \left(a_{3}\right) \\
& \left.+\cdots-\cos \left(a_{11}\right)+\cos \left(a_{12}\right)\right),
\end{aligned}
$$




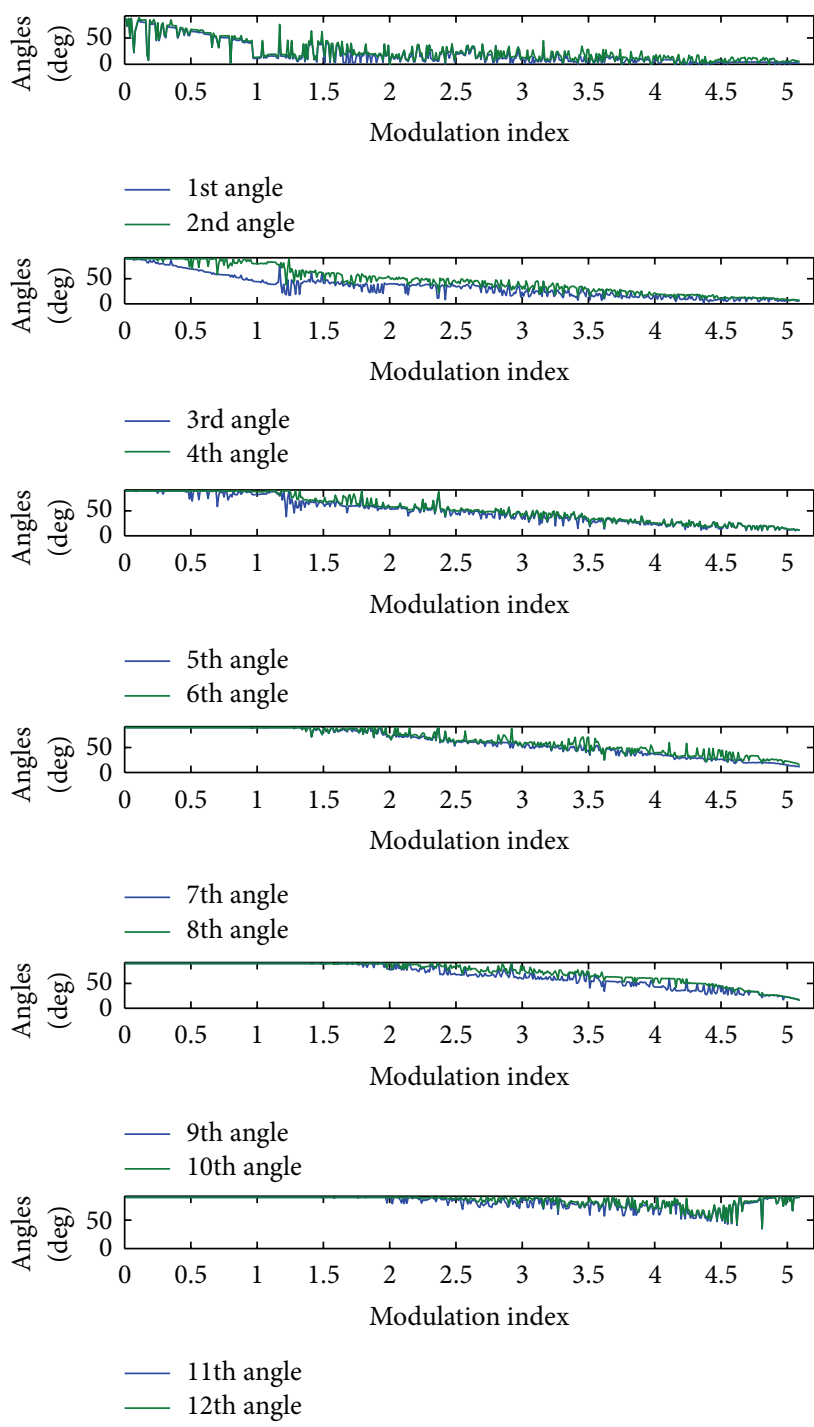

(a)

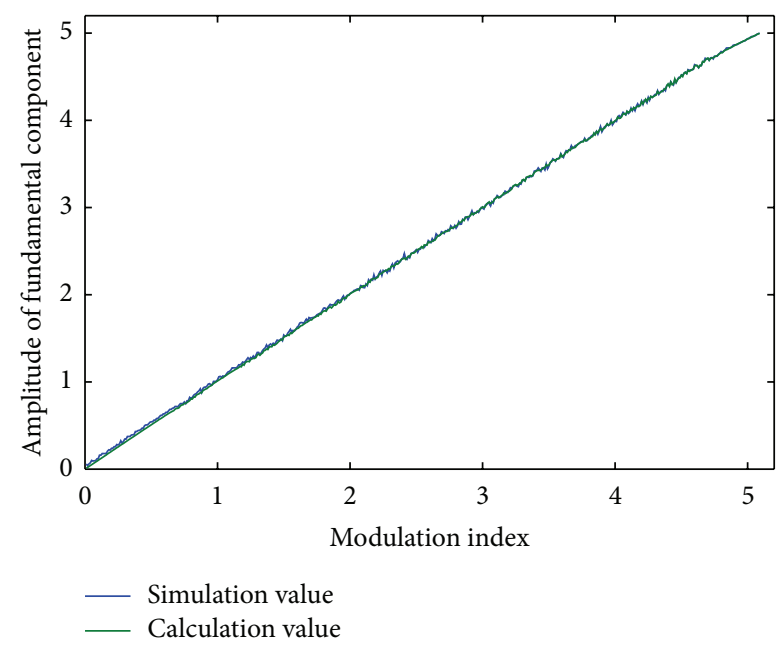

(b)

FIGURE 3: (a) Optimal switching angles versus modulation index. (b) amplitude of the fundamental component versus modulation index.

$$
\begin{gathered}
\left(V_{a}\right)_{5}=\frac{4}{5 \pi} V_{\mathrm{dc}}\left(\cos \left(5 a_{1}\right)-\cos \left(5 a_{2}\right)+\cos \left(5 a_{3}\right)\right. \\
\left.+\cdots-\cos \left(5 a_{11}\right)+\cos \left(5 a_{12}\right)\right) \\
\vdots \\
\left(V_{a}\right)_{35}=\frac{4}{35 \pi} V_{\mathrm{dc}}\left(\cos \left(35 a_{1}\right)-\cos \left(35 a_{2}\right)+\cos \left(35 a_{3}\right)\right. \\
\left.+\cdots-\cos \left(35 a_{11}\right)+\cos \left(35 a_{12}\right)\right)
\end{gathered}
$$

Satisfying the fundamental component and eliminating undesired harmonics in the same time is considered as an objective function.

Given the previous descriptions, the objective function is given by the following expression:

$$
\begin{aligned}
& \text { objective: }\left(100 \frac{V_{1}^{*}-V_{1}}{V_{1}^{*}}\right)^{4}+\sum_{i=2}^{K S} \frac{1}{h_{i}}\left(50 \frac{V_{h_{i}}}{V_{1}}\right)^{2} \text {, } \\
& i=1,2, \ldots, K S, \\
& V_{1}^{*}=\frac{V_{1}}{V_{\mathrm{dc}}},
\end{aligned}
$$




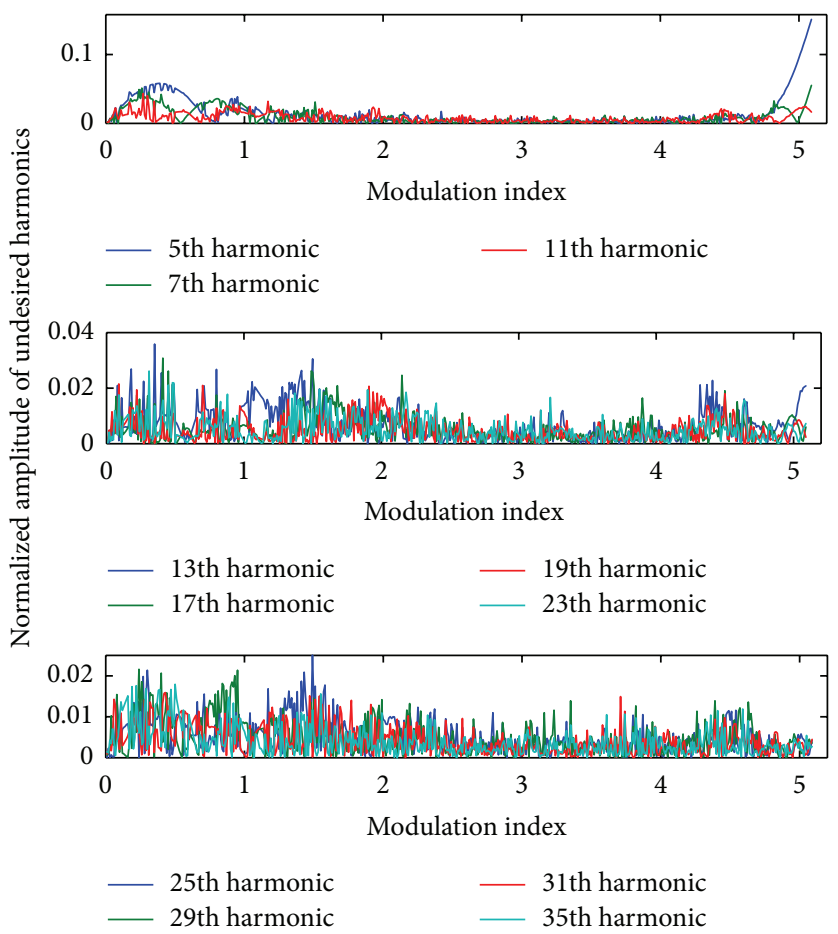

FIGURE 4: The normalized amplitude of undesired harmonics $\left(V_{h} / 4 V_{\mathrm{dc}}\right)$ versus modulation index.

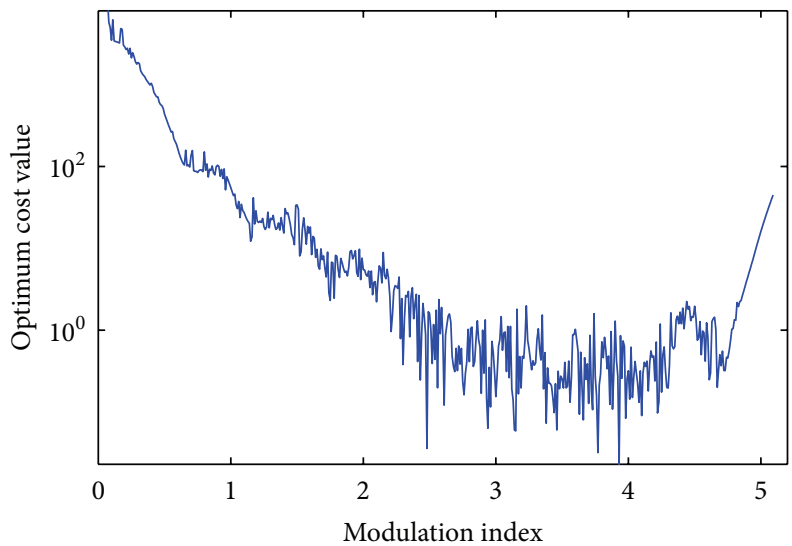

Figure 5: The optimum value of cost function versus modulation index.

where $V_{1}^{*}$ is the possible normalized amplitude of desired fundamental component which is denominated as the modulation index for this objective function varies from zero to $(4 / \pi) s, K S$ is the number of switching angles, and $h_{i}$ is the order of $i$ th viable harmonic at the output of a three-phase multilevel inverter, for example, $h_{2}=5, h_{3}=7, \ldots$ and $h_{12}=$ 35. If the fundamental component transgresses its set point by more than $1 \%$, the first term of (6) defines it by a power of 4 . Because of using the power of 4 , corresponding penalties for any digressions under $1 \%$ get a negligible value. The second term of (6) neglects harmonics under $2 \%$ of fundamental component. But, when any harmonic exceeds this limit, the objective function is subject to a penalty by power of 2 .
Finally, each harmonic ratio is weighted by inverse of its harmonic order, that is, $1 / h_{i}$. With this weighting method, reducing the low-order harmonics gets higher importance. The switching angles which are the solutions of aforementioned objective function must gratify the following basic constraint:

$$
0 \leq a_{1} \leq \cdots \leq a_{12} \leq \frac{\pi}{2} .
$$

In this paper imperialist competitive algorithm is used to solve the defined problem. Imperialist competitive algorithm is a recently developed evolutionary optimization method which is inspired by imperialist competition. Like other evolutionary algorithm, to solve a problem, it requires an initial 


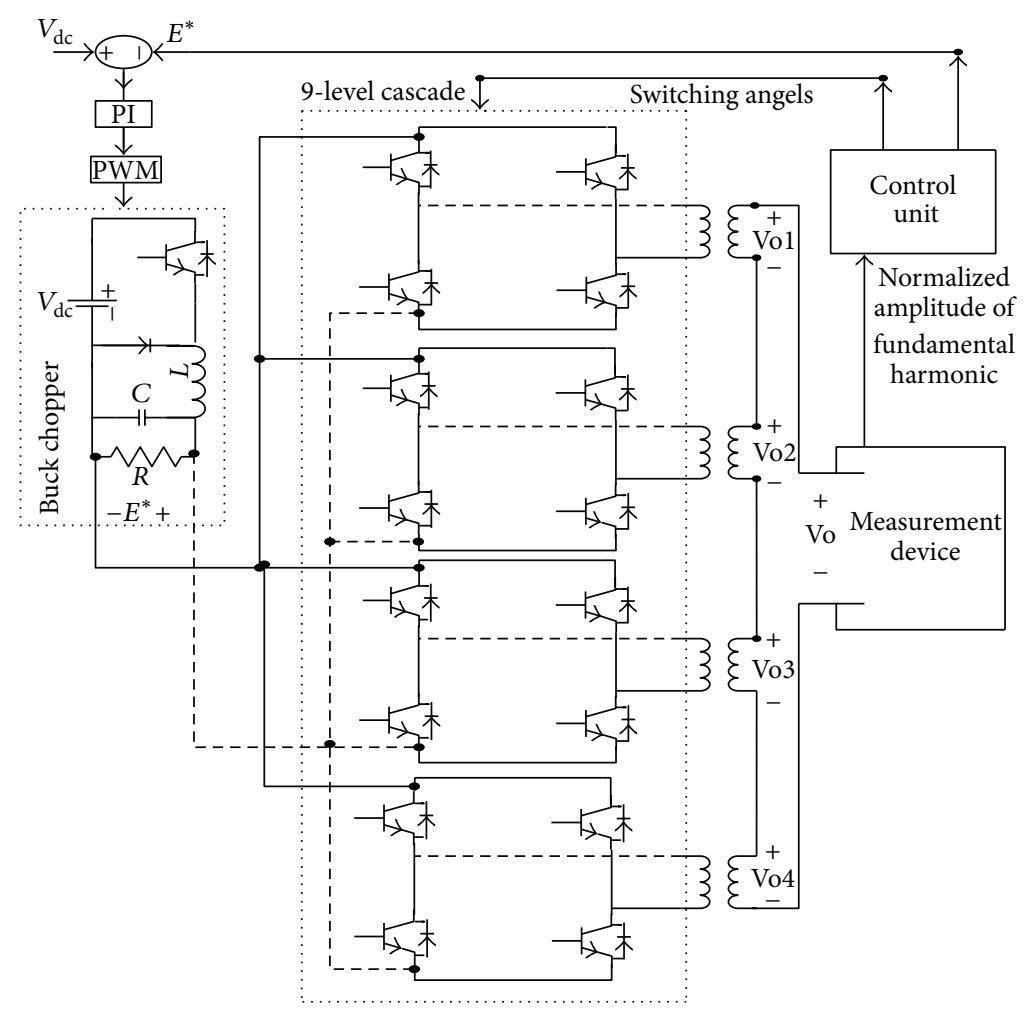

FIGURE 6: The overall system consists of the 9-level cascade inverter, the buck converter, and the required control units.

population in which finally one of them will be the proper solution of aforementioned problem. Initial population is the sum of countries which is divided into imperialists and colonies which together form empires. One imperialist with relevant colonies creates an empire. Based on assimilation policy, the colonies start moving toward their relevant imperialist state while improving their economy, culture, political, and social situation. The total power of an empire is the sum of imperialist power and the percentage of relevant colonies power. During the mentioned competition, all empires attempt to take possession of colonies of other empires until weak empires collapse and just one most powerful empire exists which the Imperialist and the relevant colonies have the same value of cost function. So, the state with most powerful empire is the convergence point of ICA. Figure 2 depicts the flowchart of ICA. With regard to the concept of SHE, variables are the switching angles of the 9-level inverter.

\section{Simulation Results}

The SHE method is applied to the nine-level inverter and the results are obtained. The corresponding optimum switching angles versus the modulation index are represented in Figure 3(a).

Figure 3(b) shows the amplitude of the fundamental component versus modulation index. The normalized amplitude of undesired harmonics $\left(V_{h} / 4 V_{\mathrm{dc}}\right)$ versus modulation index is plotted in Figure 4. It can be seen from this figure that the amplitude of undesired harmonics has very low values and so the presented method can properly eliminate the selected harmonic components. Figure 5 illustrates the amount of cost function with respect to the modulation index.

The obtained solutions for the switching angles and the corresponding cost values are presented in Figures 3(a) and 5, respectively. From the cost values shown in Figure 5, it is apparent that the ICA algorithm is effectively able to find the optimum switching angles to meet the objective function which aims to achieve the fundamental component and suppress the undesired harmonics. As illustrated before, there is no possibility to meet the objective function in all ranges where SHE is applied. As shown in Figure 5 it is clear that for some ranges of modulation index the value of cost function is low and it means that all proposed undesired harmonics get nearly to zero. The ranges of $M$ with high values of cost function mean that the values of harmonic components are nonzero, and so the equations cannot be solved and there is no solution. Mostly in the whole ranges of modulation index, shown in Figure 3, the fundamental component is kept close to the desired value. Based on presented simulation results it can be seen that there are some regions of modulation index in which the optimum value of switching angles do not exist. To overcome this problem a DC/DC inverter is used in DC link of inverter. This converter controls the DC link voltage of inverter, $E^{*}$. Figure 6 shows the overall systems content, includes the 9-level cascade inverter, the buck converter, and the required control unit to coordinate switching pattern with the input DC voltage of the 9-level cascade inverter.

It can be seen from Figure 5 that there are not proper solutions for switching angles in all modulation indexes. Therefore, when the fundamental harmonic amplitude is 

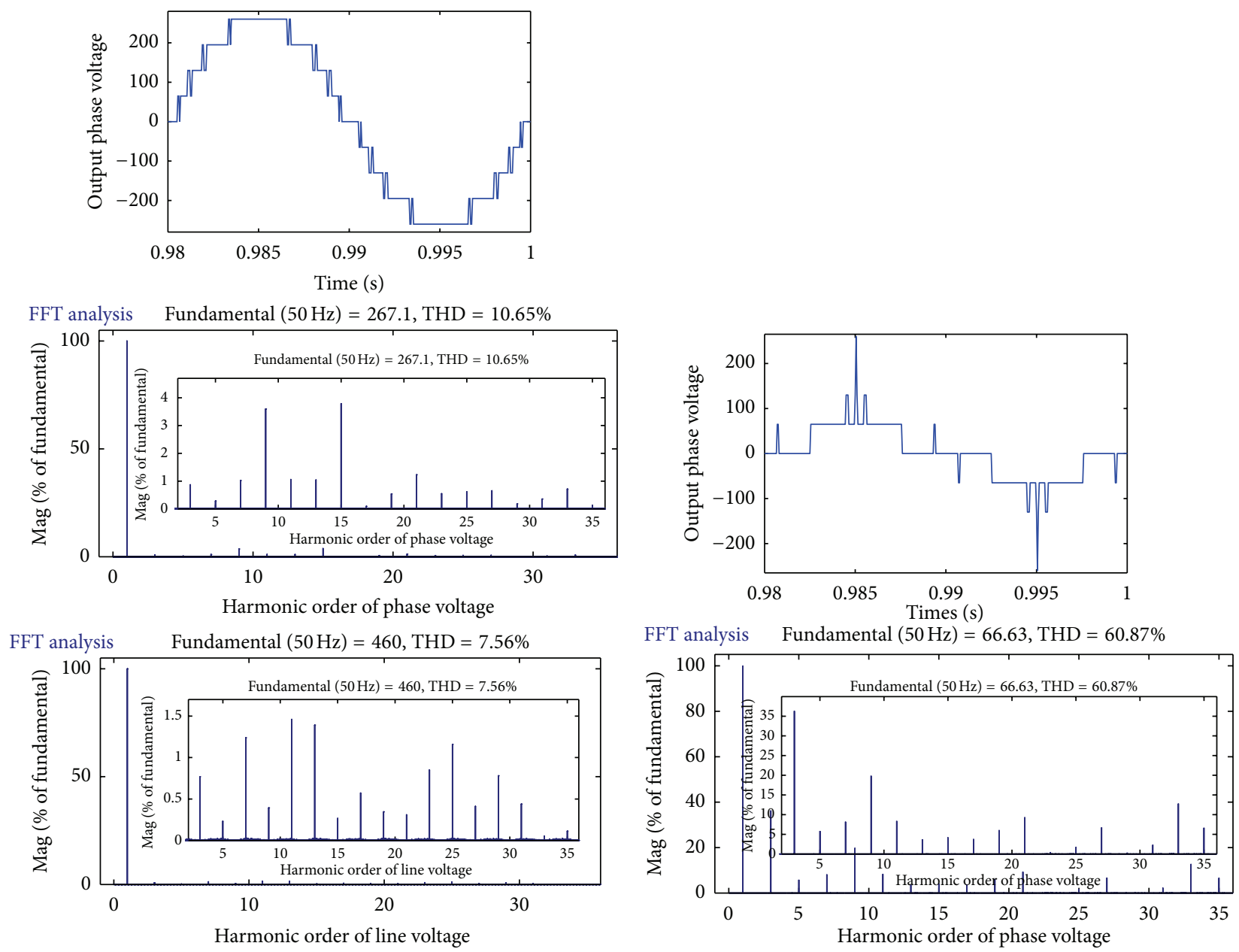

(a) $M=4.12$ and $E^{*}=65^{\mathrm{v}}$

(b) $M=1$ and $E^{*}=65^{\mathrm{v}}$
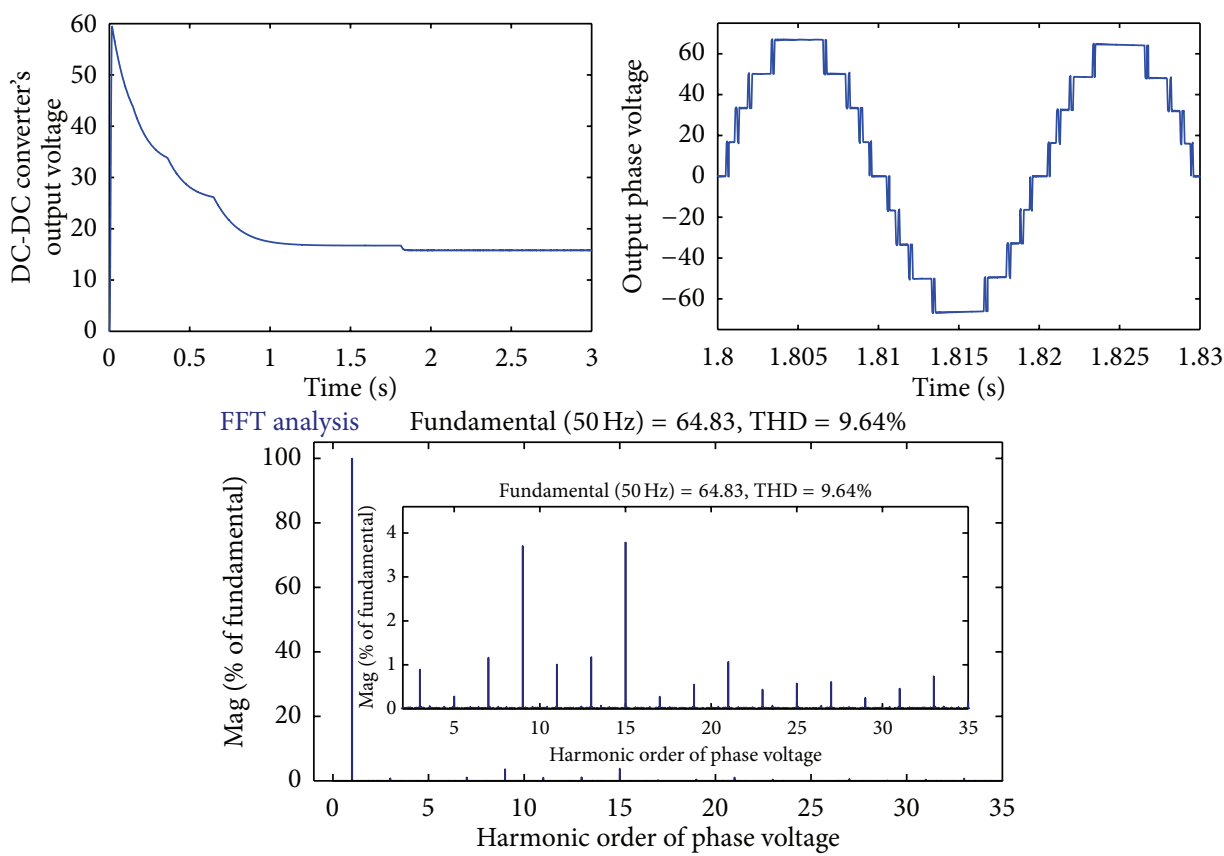

(c) $M=1$ and $E^{*}=15.8^{\mathrm{v}}$

FIGURE 7: The output voltage waveform of inverter and the harmonic content for (a) $M=4.12, E^{*}=65^{\mathrm{v}}$, (b) $M=1, E^{*}=65^{\mathrm{v}}$, and (c) $M=1$, $E^{*}=15.8^{\mathrm{v}}$. 


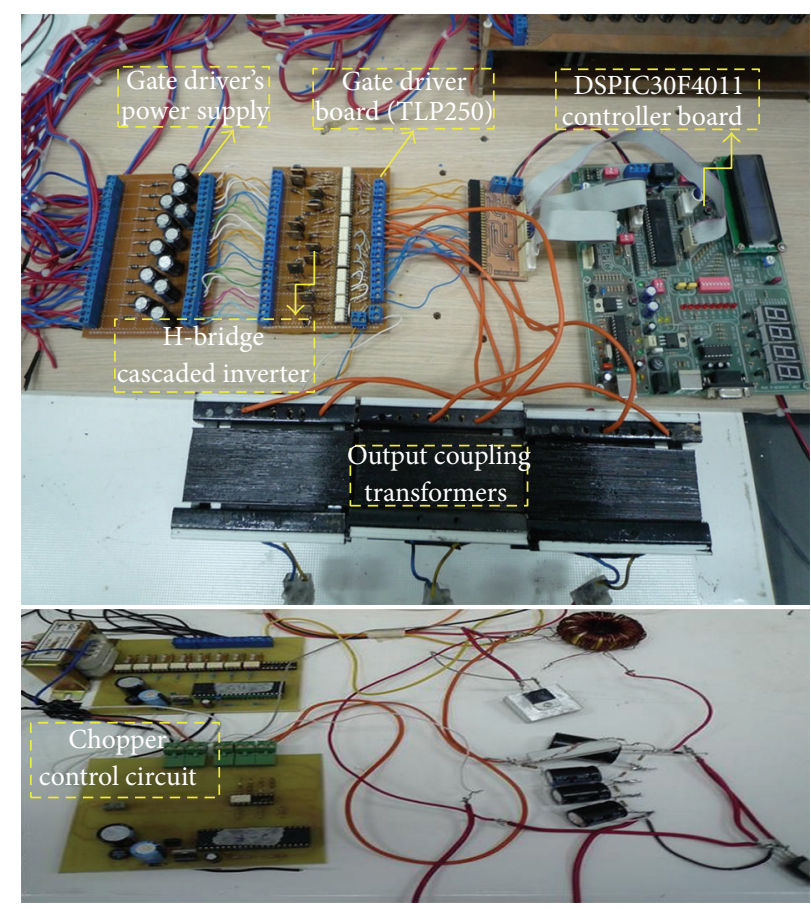

FIGURE 8: The implemented prototype setup.

TABLE 1: Switching principle $\left(V_{\mathrm{dc}}=65 \mathrm{v}=1 \mathrm{pu}\right)$.

\begin{tabular}{|c|c|c|c|c|c|c|c|c|c|c|c|c|c|}
\hline$M$ & $a_{1}^{\prime}\left({ }^{\circ}\right)$ & $a_{2}^{\prime}\left({ }^{\circ}\right)$ & $a_{3}^{\prime}\left({ }^{\circ}\right)$ & $a_{4}^{\prime}\left(^{\circ}\right)$ & $a_{5}^{\prime}\left({ }^{\circ}\right)$ & $a_{6}^{\prime}\left({ }^{\circ}\right)$ & $a_{7}^{\prime}\left({ }^{\circ}\right)$ & $a_{8}^{\prime}\left({ }^{\circ}\right)$ & $a_{9}^{\prime}\left({ }^{\circ}\right)$ & $a_{10}^{\prime}\left({ }^{\circ}\right)$ & $a_{11}^{\prime}\left({ }^{\circ}\right)$ & $a_{12}^{\prime}\left({ }^{\circ}\right)$ & $\begin{array}{c}E^{*}(\mathrm{pu}) \\
V_{\text {base }}=65 \mathrm{~V}\end{array}$ \\
\hline 4.12 & 8.496 & 10.033 & 10.886 & 18.183 & 21.298 & 23.353 & 32.775 & 35.01 & 37.771 & 59.203 & 61.174 & 62.1 & $1 \mathrm{pu}$ \\
\hline 1 & 11.241 & 13.284 & 44.187 & 80.088 & 82.678 & 88.571 & 89.084 & 89.085 & 89.996 & 89.997 & 89.998 & 89.999 & $1 \mathrm{pu}$ \\
\hline 1 & 8.496 & 10.033 & 10.886 & 18.183 & 21.298 & 23.353 & 32.775 & 35.01 & 37.771 & 59.203 & 61.174 & 62.1 & $0.2429 \mathrm{pu}$ \\
\hline
\end{tabular}

TABLE 2: The parameters of the implemented circuit.

\begin{tabular}{lc}
\hline Switches & IRF $840,500 \mathrm{v}, 8 \mathrm{~A}$ \\
Gate driver & TLP 250 \\
$L$ & $10 \mathrm{mH}$ \\
$C$ & $0.1 \mathrm{mF}$ \\
$R$ & $5000 \mathrm{ohm}$ \\
$V_{\mathrm{dc}}$ & $65 \mathrm{v}$ \\
\hline
\end{tabular}

in region without solution, the control unit sends a signal, includes required input DC voltage, $E^{* \mathrm{NEW}}$, of the inverter, to the DC-DC converter, and modifies the exciting switching pattern based on new DC link voltage and sends it to the inverter. Following expression is the basic constraints must be gratified by control unit:

$$
\begin{gathered}
{\left[\left(\cos \left(a_{1}\right)-\cos \left(a_{2}\right)+\cos \left(a_{3}\right)+\cos \left(a_{4}\right)-\cdots\right.\right.} \\
\left.\left.-\cos \left(a_{11}\right)+\cos \left(a_{12}\right)\right)\right]^{\mathrm{NEW}} * E^{* \mathrm{NEW}}=\frac{M}{(4 / \pi)} \\
0.2 V_{\mathrm{dc}} \leq E^{* \mathrm{NEW}} \leq V_{\mathrm{dc}}
\end{gathered}
$$

The new switching pattern is the one of several optimum switching angles that are collected in a look-up table obtained from SHE. The moderate capability of chopper on strengthening and castrating the input voltage is indicated as a constraint in (10). For a case study the output voltage waveforms for $M=1$ and $M=4.12$ with $E^{*}=1^{\mathrm{pu}}$ are plotted in Figure 9. To enhance the harmonic spectra for $M=1$, the controlling of DC-DC converter output voltage and the switching angles of the inverter are required. So to satisfy $M=1$ the switching angles of $M=4.12$ with appropriate voltage $E^{*}=0.2429^{\mathrm{pu}}$ are required. The simulation results of this case are presented in Figure 9(c). The switching angles (degree) and the required voltage value are listed in Table 1 .

As shown in Figures 7(a)-7(c) the amplitude of fundamental components is almost close to the modulation index. It can be seen from Figure 7(a) that the amplitude of undesired harmonics is negligible. However, Figure 7(b) shows the high value in THD of output voltage. To reduce the THD value, as described before, DC-DC converter is used to control the DC link of the inverter which causes a significant reduction in output THD value Figure $7(\mathrm{c})$.

\section{Experimental Results}

To confirm the validation and effectiveness of presented method in this paper, an experimental prototype of a 

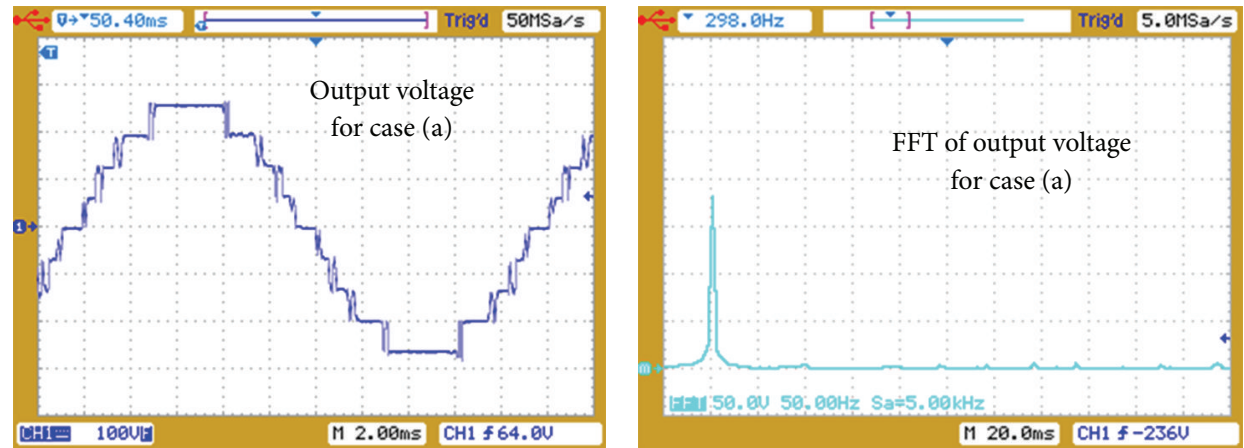

(a) $M=4.12$ and $E^{*}=65^{\mathrm{v}}$
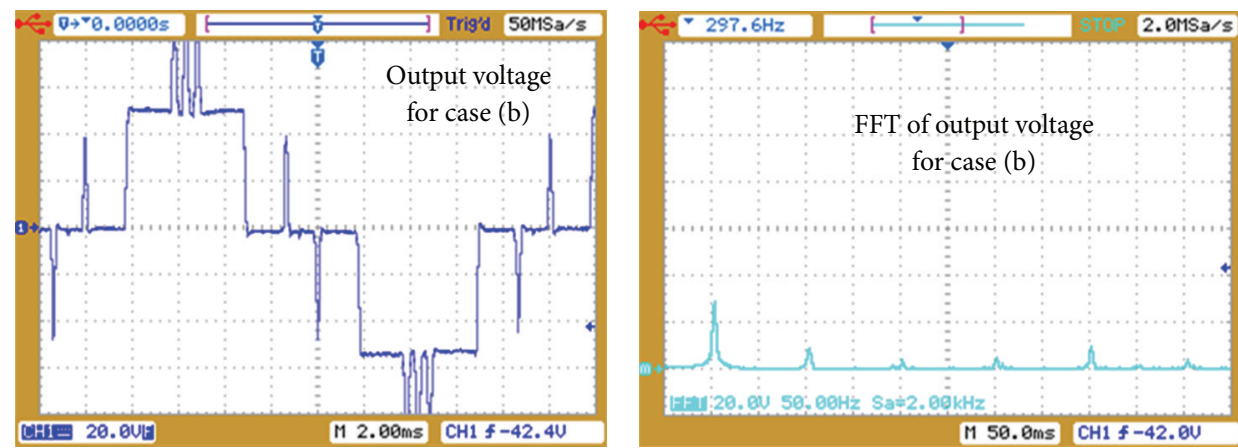

(b) $M=1$ and $E^{*}=65^{\mathrm{v}}$
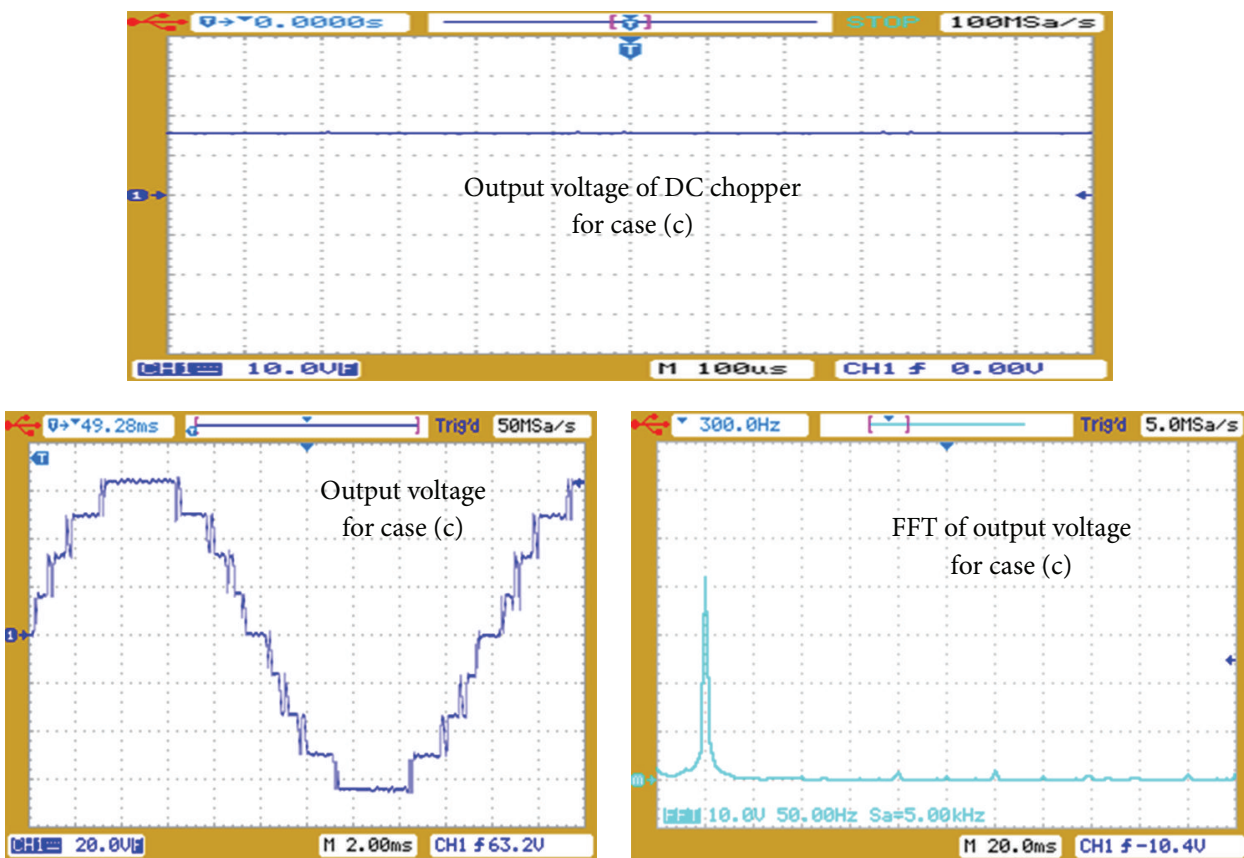

(c) $M=1, E^{*}=15.8^{\mathrm{v}}$

FIGURE 9: Experimental results of inverter output voltage and the harmonic content for (a) $M=4.12, E^{*}=65^{\mathrm{v}}$, (b) $M=1, E^{*}=65^{\mathrm{v}}$, and (c) $M=1, E^{*}=15.8^{\mathrm{v}}$.

single-phase nine-level cascaded inverter with a buck converter in DC side of inverter has been implemented. Figure 8 shows the implemented prototype. Parameters of implemented circuit are given in Table 2. Figure 9 depicts the experimental results of implemented circuit. It can be seen from Figures 9(a)-9(c) that the experimental voltage waveforms are more similar to simulation results and the harmonic spectra of mentioned waveforms validate the implemented methods efficiency. Also, in Figure 9(c) the output voltage of DC-DC converter is shown. It can be seen from 
this figure that the DC-DC converter can properly track the reference voltage.

It can be seen from Figures 9(a)-9(c) that the obtained voltage waveforms are more similar to simulation results and the harmonic spectra of mentioned waveforms validate the implemented methods efficiency. Also, in Figure 9(c) the target value of output voltage of DC-DC converter is obtained.

\section{Conclusion}

An ICA algorithm has been suggested to determine the optimum switching angles of a 9-level inverter. This algorithm has been successfully exerted to the SHE-PWM problem with larger number of switching angles, where other traditional methods cannot solve it. Simulation and experimental results are provided for a 9-level inverter to confirm the accuracy of suggested method. Results show that the defined undesired harmonics have been successfully minimized at the output voltage waveform. Also to develop the sufficiency of the SHE to all range of modulation index a DC-DC converter is used to control the input DC voltage of the inverter which dramatically improves the SHE effectiveness on eliminating the undesired harmonics.

\section{References}

[1] W. Song and A. Q. Huang, "Fault-tolerant design and control strategy for cascaded $\mathrm{H}$-bridge multilevel converter-based STATCOM," IEEE Transactions on Industrial Electronics, vol. 57, no. 8, pp. 2700-2708, 2010.

[2] N. Farokhnia, S. H. Fathi, and H. Toodeji, "Direct nonlinear control for individual DC voltage balancing in cascaded multilevel DSTATCOM," in Proceedings of the International Conference on Electric Power and Energy Conversion Systems (EPECS '09), pp. 1-8, November 2009.

[3] L. M. Tolbert, F. Z. Peng, and T. G. Habetler, "Multilevel converters for large electric drives," IEEE Transactions on Industry Applications, vol. 35, no. 1, pp. 36-44, 1999.

[4] V. Naumanen, J. Korhonen, P. Silventoinen, and J. Pyrhönen, "Multilevel modulation method for mitigation of high du/dtoriginated oscillating overvoltages at motor terminals," IET Power Electronics, vol. 4, no. 1, pp. 29-38, 2011.

[5] E. Ozdemir, S. Ozdemir, and L. M. Tolbert, "Fundamental-frequency-modulated six-level diode-clamped multilevel inverter for three-phase stand-alone photovoltaic system," IEEE Transactions on Industrial Electronics, vol. 56, no. 11, pp. 4407-4415, 2009.

[6] E. Villanueva, P. Correa, J. Rodriguez, and M. Pacas, "Control of a single-phase cascaded H-bridge multilevel inverter for gridconnected photovoltaic systems," IEEE Transactions on Industrial Electronics, vol. 56, no. 11, pp. 4399-4406, 2009.

[7] D. Ahmadi, K. Zou, C. Li, Y. Huang, and J. Wang, "A universal selective harmonic elimination method for high-power inverters," IEEE Transactions on Power Electronics, vol. 26, no. 10, pp. 2743-2752, 2011.

[8] M. R. Banaei and E. Salary, "New multilevel inverter with reduction of switches and gate driver," Energy Conversion and Management, vol. 52, no. 2, pp. 1129-1136, 2011.

[9] L. M. Tolbert, J. N. Chiasson, Z. Du, and K. J. McKenzie, "Elimination of harmonics in a multilevel converter with nonequal
DC sources," IEEE Transactions on Industry Applications, vol. 41, no. 1, pp. 75-82, 2005.

[10] J. N. Chiasson, L. M. Tolbert, K. J. McKenzie, and Z. Du, "A unified approach to solving the harmonic elimination equations in multilevel converters," IEEE Transactions on Power Electronics, vol. 19, no. 2, pp. 478-490, 2004.

[11] D. Ahmadi, K. Zou, and J. Wang, "Weight orientedoptimal PWM in low modulation indexes for multilevel inverters with unbalanced DC sources," in Proceedings of the 25th Annual IEEE Applied Power Electronics Conference and Exposition (APEC '10), pp. 1038-1042, February 2010.

[12] M. Tarafdar Hagh, H. Taghizadeh, and K. Razi, "Harmonic minimization in multilevel inverters using modified species-based particle swarm optimization," IEEE Transactions on Power Electronics, vol. 24, no. 10, pp. 2259-2267, 2009.

[13] A. K. Al-Othman and T. H. Abdelhamid, "Elimination of harmonics in multilevel inverters with non-equal dc sources using PSO," Energy Conversion and Management, vol. 50, no. 3, pp. 756-764, 2009.

[14] H. Taghizadeh and M. Tarafdar Hagh, "Harmonic elimination of cascade multilevel inverters with nonequal dc sources using particle swarm optimization," IEEE Transactions on Industrial Electronics, vol. 57, no. 11, pp. 3678-3684, 2010.

[15] N. Yousefpoor, S. H. Fathi, N. Farokhnia, and H. A. Abyaneh, "THD minimization applied directly on the line-to-line voltage of multilevel inverters," IEEE Transactions on Industrial Electronics, vol. 59, no. 1, pp. 373-380, 2012.

[16] V.-T. Phan and H.-H. Lee, "Control strategy for harmonic elimination in stand-alone DFIG applications with nonlinear loads," IEEE Transactions on Power Electronics, vol. 26, no. 9, pp. 26622675, 2011.

[17] Z. Du, L. M. Tolbert, J. N. Chiasson, and B. Ozpineci, "Reduced switching-frequency active harmonic elimination for multilevel converters," IEEE Transactions on Industrial Electronics, vol. 55, no. 4, pp. 1761-1770, 2008.

[18] R. N. Ray, D. Chatterjee, and S. K. Goswami, "Harmonics elimination in a multilevel inverter using the particle swarm optimisation technique," IET Power Electronics, vol. 2, no. 6, pp. 646652, 2009.

[19] A. Kavousi, B. Vahidi, R. Salehi, M. K. Bakhshizadeh, N. Farokhnia, and S. H. Fathi, "Application of the bee algorithm for selective harmonic elimination strategy in multilevel inverters," IEEE Transactions on Power Electronics, vol. 27, no. 4, pp. 16891696, 2012.

[20] L. Li, D. Czarkowski, Y. Liu, and P. Pillay, "Multilevel selective harmonic elimination PWM technique in series-connected voltage inverters," IEEE Transactions on Industry Applications, vol. 36, no. 1, pp. 160-170, 2000.

[21] F.-S. Shyu and Y.-S. Lai, "Virtual stage pulse-width modulation technique for multilevel inverter/converter," IEEE Transactions on Power Electronics, vol. 17, no. 3, pp. 332-341, 2002.

[22] B. Ozpineci, L. M. Tolbert, and J. N. Chiasson, "Harmonic optimization of multilevel converters using genetic algorithms," IEEE Power Electronics Letters, vol. 3, no. 3, pp. 92-95, 2005.

[23] K. J. McKenzie, Eliminating harmonics in a cascaded H-bridges multilevel inverter using resultant theory, symmetric polynomials, and power sums [M.S. thesis], University of Tennessee, Chattanooga, Tenn, USA, 2004. 

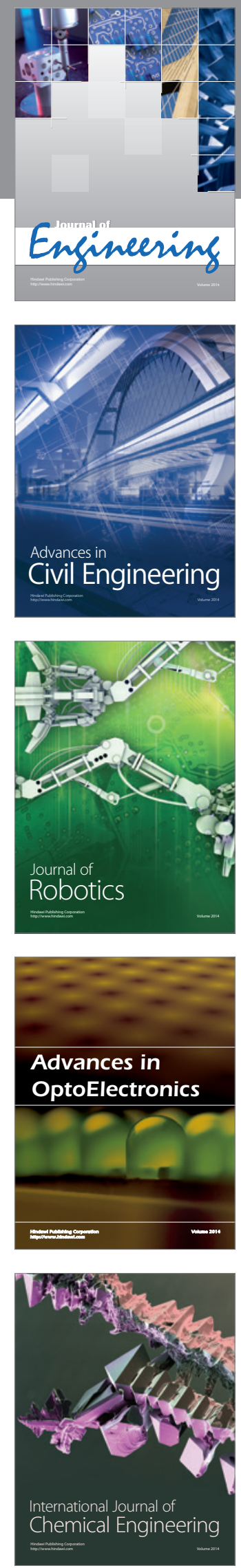

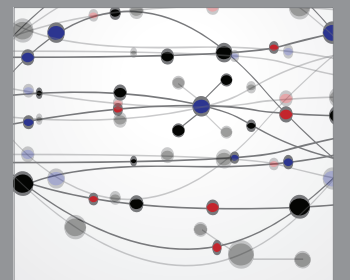

The Scientific World Journal
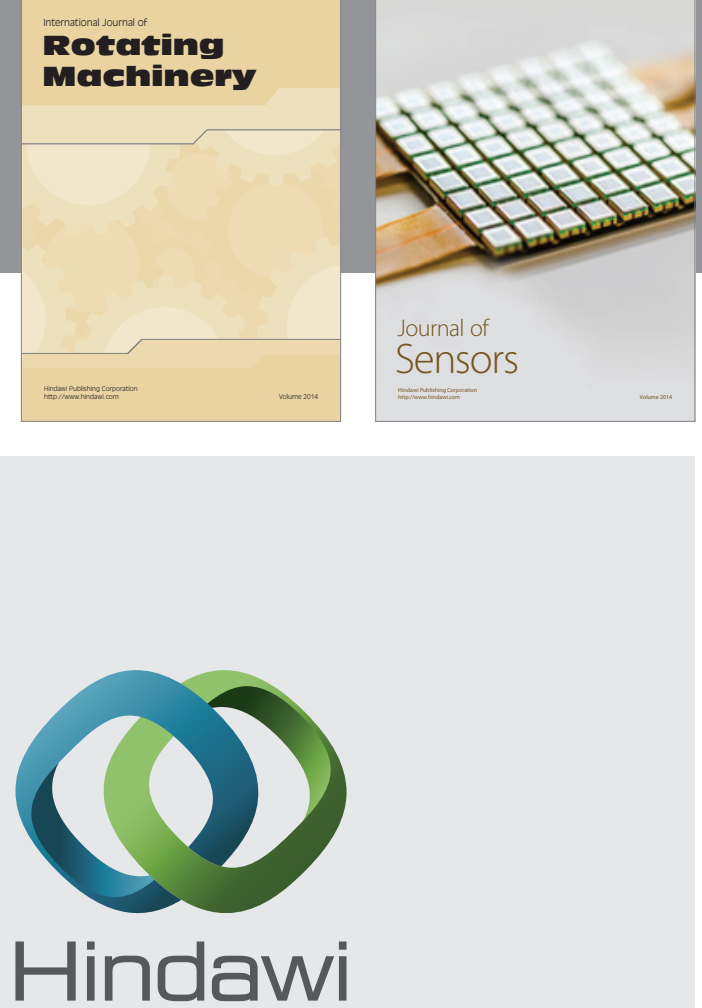

Submit your manuscripts at http://www.hindawi.com
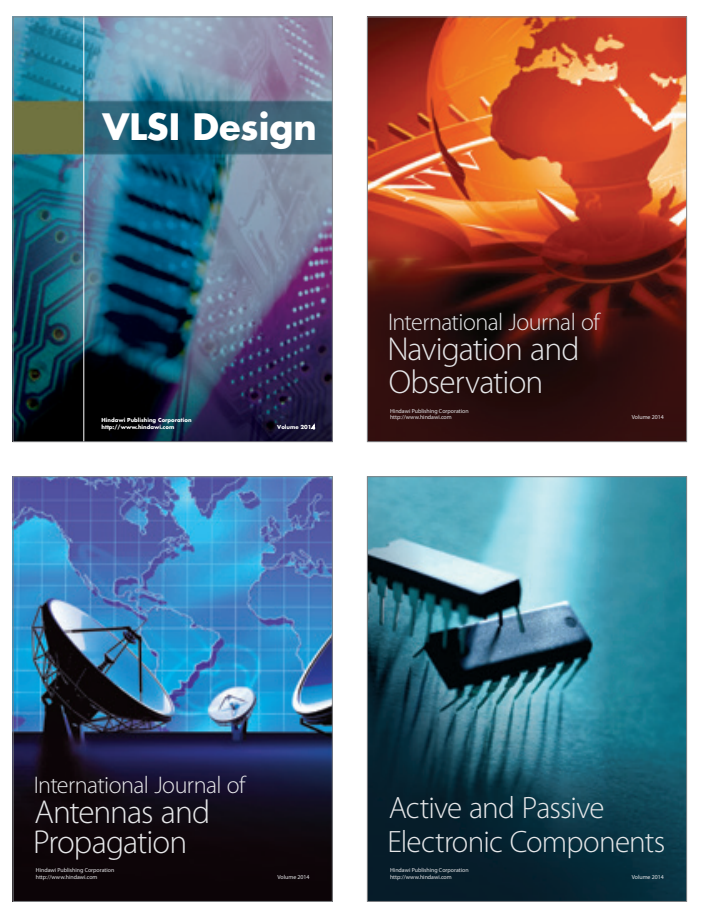
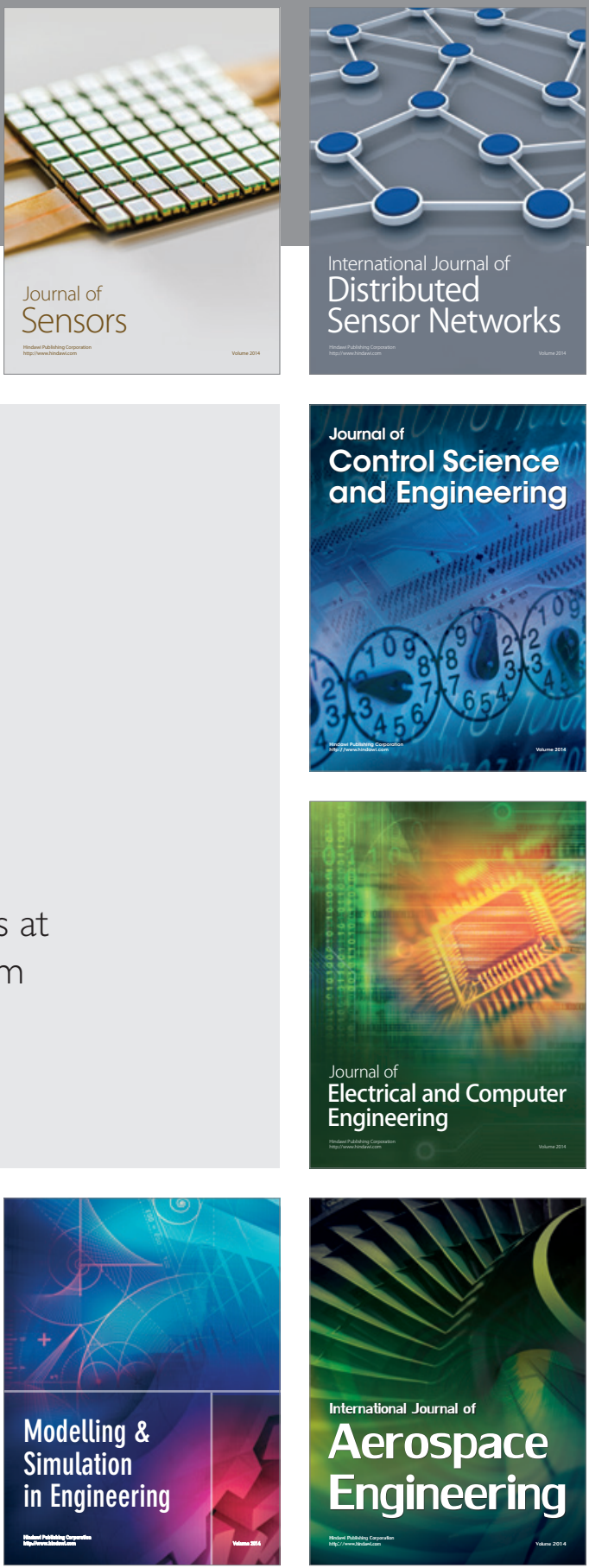

Journal of

Control Science

and Engineering
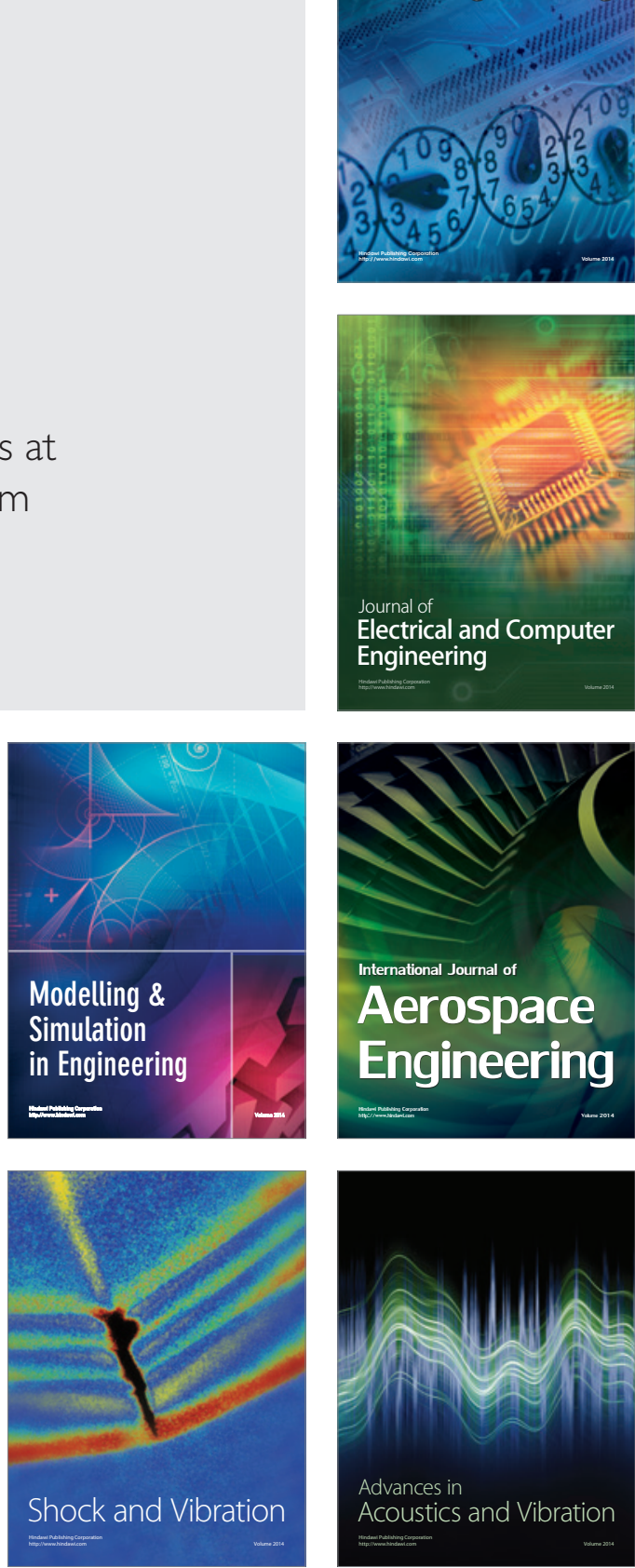